REGARDS

SUR LECONOMIE ALLEMANDE

BULLETIN ECONOMIQUE DU CIRAC
Regards sur l'économie allemande

Bulletin économique du CIRAC

$72 \mid 2005$

Varia

\title{
IG Metall pour un Etat social plus équitable
}

\section{Wolfgang Schroeder}

Traducteur : Isabelle Bourgeois

\section{OpenEdition}

\section{Journals}

Édition électronique

URL : http://journals.openedition.org/rea/250

DOI : $10.4000 /$ rea. 250

ISBN : 978-2-8218-0840-9

ISSN : 1965-0787

Éditeur

CIRAC

Édition imprimée

Date de publication : 1 juillet 2005

Pagination : 17-22

ISSN : 1156-8992

\section{Référence électronique}

Wolfgang Schroeder, "IG Metall pour un Etat social plus équitable », Regards sur l'économie allemande

[En ligne], 72 I juillet 2005, mis en ligne le 06 mai 2008, consulté le 14 novembre 2019. URL : http:// journals.openedition.org/rea/250 ; DOI : 10.4000/rea.250 


\section{IG Metall pour un Etat social plus équitable}

\section{Wolfgang Schroeder}

« L'Etat social doit évoluer. Le statu quo n'est plus tenable : il ne permet ni de répondre aux défis actuels ni encore moins d'assurer la pérennité du système ». C'est en ces termes que W. Schrœder, responsable du Département Politique sociale d'IG Metall, nous expliquait dans un entretien (voir REA 69/04) la nouvelle orientation de son syndicat dans le champ social. A cet effet, IG Metall (2,4 millions d'adhérents) avait organisé à Berlin, les 6 et 7 avril 2005, un Congrès sur l'Etat social rassemblant quelque 600 participants. Ce Sozialstaatskongress, premier du genre, a été l'occasion de dresser le bilan des mutations structurelles intervenues depuis les années 1970 et de leur impact sur l'avenir du modèle social allemand. Lieu d'analyse et de réflexion collectives, il n'avait pas pour objectif de définir un programme d'action syndicale.

Le pivot des réflexions était le constat suivant: la politique conventionnelle en matière de salaires et de temps de travail (Tarifpolitik) s'est transmuée au fil du temps en « une sorte de réserve de flexibilité pour la politique menée en matière de protection sociale », comme le formule le syndicat. En d'autres termes, la Tarifpolitik a changé d'approche : elle tend désormais à intégrer la rémunération indirecte au détriment de la rémunération directe, comme on peut l'observer par exemple dans la hausse du temps de travail sans compensation salariale (voir REA 68/04) mais avec, en contrepartie, une offre de prestations de protection sociale (par exemple l'accès aux régimes de retraites d'entreprise ; voir REA 70/05.). L'orientation de la politique contractuelle des partenaires sociaux a subi une profonde inflexion : elle intègre désormais le facteur des coûts salariaux annexes.

C'est là une réponse aux transformations du contexte dans lequel évolue le modèle social allemand (vieillissement démographique, mutations du travail, globalisation, européanisation). L'action menée sur le seul champ des revenus salariaux atteint cependant ses limites et appelle une approche plus large. Le premier syndicat allemand à thématiser cette inflexion est IG Metall, dont le Sozialstaatskongress visait à sensibiliser ses adhérents à une refondation de son action revendicative autour d'une approche désormais globale du champ social. Nous avons demandé à W. Schrœder, dont le département a piloté le Congrès, de nous exposer les positions développées par son syndicat. Voici, résumées en 11 points, les orientations officielles d'IG Metall pour une réforme du modèle social allemand et la réalisation d'une Europe sociale. (IB)

\section{Non pas défendre le statu quo, mais faire évoluer le modèle solidaire}

L'Etat social allemand subit une transformation rapide et profonde. Les mutations affectent ses bases statutaires, son financement paritaire et la contribution équitable de tous. Or ces trois piliers de l'Etat social allemand ont été mis à mal au cours des dernières années. Les causes des mutations de l'Etat social sont nombreuses. Aux difficultés économiques générées par la globalisation et l'Unité allemande s'ajoutent plus particulièrement les transformations de la société liées aux mutations du travail, à l'évolution des conditions de vie, ainsi qu'au vieillissement démographique. La réponse d'IG Metall à tous ces changements est univoque: mener une politique du statu quo ne permet pas de construire

"Le statu quo ne permet pas de construire l'avenir » 
l'avenir des systèmes de prévoyance et de protection sociales. II nous faut au contraire refonder l'Etat providence en le dotant d'instruments nouveaux et en modifiant son financement.

\section{"Avoir le courage de l'équité "}

Dans le cadre de son « Congrès sur l'Etat social » des 6 et 7 avril 2005 à Berlin, IG Metall avait élaboré un discussion paper intitulé Mut zur Gerechtigkeit : « Avoir le courage de l'équité ». Ce texte, disponible en ligne, détaille les mutations structurelles du contexte des activités et les défis qu'elles posent à un syndicalisme aujourd'hui en porte-à-faux du fait des reconfigurations de branches. Pour IG Metall, il s'agit désormais, « dans l'intérêt de nos membres comme dans celui de notre crédibilité, [de] formuler une position autonome » dans le débat lié aux réformes sociales allemandes, ainsi que l'expliquait son premier président, Jürgen Peters, en octobre 2004. En effet, les syndicats allemands ne sont pas seulement responsables de la fixation des salaires ou de la durée du temps de travail, mais ils sont également co-gestionnaires des caisses de retraite et d'assurance maladie, comme de l'Agence fédérale pour l'emploi qui gère le système d'indemnisation chômage et les mesures actives en faveur de l'emploi. C'est donc en tant qu'acteur institutionnel (et doté de l'autonomie administrative) du système bismarckien qu'IG Metall cherche aujourd'hui à co-définir les options de modernisation d'un modèle social allemand qui, s'il se montre "généreux au plan des transferts, n'œuvre guère pour la justice sociale », comme nous le confiait W. Schrœder lors d'un entretien (voir REA 69/04).

IG Metall place ses propositions sur l'orientation des réformes dans une approche globale, macro-économique et macro-sociale, exerçant là les responsabilités que confère aux partenaires sociaux la Loi fondamentale. Conscient de l'impératif des réformes, il n'en concilie pas moins cette approche avec la culture d'un mouvement syndical traditionnellement proche des valeurs sociales-démocrates. Or celles-ci, comme l'a révélé le cap des réformes du gouvernement Schröder, ont évolué avec les mutations du contexte. Les positions actuelles d'IG Metall sur les retraites, la santé et l'emploi sont révélatrices à cet égard.

Retraites. IG Metall, qui salue expressément de développement de la capitalisation, s'inspire du modèle suédois où ce complément est obligatoire. Surtout, il souhaite développer le fonds de pension mutualiste propre à la métallurgie : MetallRente, créé en 2001 conjointement avec son partenaire patronal Gesamtmetall en réponse au refus des entreprises de la branche de constituer des fonds de retraite d'entreprises. Pour les y associer toutes, le syndicat souhaite rendre ce régime obligatoire.

Santé. Pour «mettre fin à la médecine de classe » et pour déconnecter le financement du facteur travail, IG Metall plaide donc doublement pour un élargissement de l'assiette du financement de l'assurance santé et pour la baisse des prélèvements. Mais s'il préconise le maintien d'un régime d'assurance légale, budgétairement consolidé, c'est pour que "les caisses publiques voient s'améliorer leur compétitivité face aux caisses privées » (W. Schrœder). II s'agit donc clairement de développer la concurrence dans ce domaine en laissant à l'assuré le libre choix de sa caisse. En ce sens, IG Metall partage l'analyse du Conseil des Sages sur l'orientation de la réforme de l'assurance maladie, formulée dans son rapport 2002/3 (voir REA 59/02).

Emploi. Considérant le travail comme la clef de l'intégration sociale, IG Metall salue expressément les réformes Hartz. Mais il en voit aussi les limites, bureaucratiques et économiques. II partage dès lors avec tous les experts, économistes et partis politiques, l'analyse selon laquelle seul un ensemble de réformes macro-économiques permettra à terme le retour de la croissance et de l'emploi. Fidèle à sa longue politique de défense de salaires élevés pour des actifs aux qualifications elles aussi généralement élevées, il met l'accent sur une hausse des qualifications et un effort de formation tout au long de la vie pour tenir compte des besoins des entreprises dans une économie du savoir naissante, sans rompre avec ses exigences en matière de niveaux salariaux.

Un rôle accru de l'entreprise dans le système de protection sociale. Dans sa politique salariale, IG Metall prend désormais en considération l'ensemble des composantes de la rétribution du travail, comme le montrent les négociations récemment conclues chez DaimlerChrysler ou VW, apportant là une contribution à la baisse des coûts salariaux annexes tout en cherchant à rééquilibrer partiellement le financement du système de protection sociale allemand. (IB)

Pour plus d'informations : www.igmetall.de/themen/sozialstaat/kongress/index.html

Rétablir la confiance

\section{Les réponses apportées jusqu'ici sont insuffisantes}

Plaider pour une refondation de l'Etat social ne signifie pas pour autant approuver les réformes mises en oeuvre ou engagées. Bien au contraire : les réformes sociales entreprises en Allemagne depuis la fin des années 1970 n'ont pas été en mesure d'assurer l'avenir de l'Etat social allemand. Aucune des mutations structurelles de la société n'a trouvé de réponse adaptée, aucun problème, aucune déficience du modèle social existant n'a reçu les remèdes requis. Pis encore, les réformes entreprises ont fini par gravement mettre à mal non seule- 
ment la fonctionnalité de l'Etat social, mais aussi, et surtout, sa légitimité. Les Allemands ont nettement perdu confiance dans l'efficience comme dans la justice de leurs systèmes de protection sociale. En conséquence, toute réforme du régime actuel doit d'abord et avant tout rétablir la confiance dans les acteurs et les dispositifs de la politique sociale - sinon, elle court à l'échec.

A mesure que les biographies professionnelles perdent leur linéarité et que les conditions de vie se modifient, les individus ont plus que jamais besoin que l'Etat social les incite à développer leur autonomie et leur responsabilité sur un marché du travail comme dans une société en profonde évolution. Voilà pourquoi prévoyance individuelle et garantie du standard de vie sont deux objectifs nullement contradictoires de la politique sociale. Ils sont par nature complémentaires. La même complémentarité caractérise la relation entre les prestations destinées à garantir un minimum vital et celles visant à assurer le maintien d'un standard de vie.

\section{Le modèle de l'Etat social démocratique : protection sociale pour tous et répartition équitable des charges}

La politique sociale d'IG Metall s'oriente par rapport au modèle d'un « Etat social démocratique, solidaire et actif ». Cette approche « reflète notre vision qui est de ne pas abandonner l'évolution de la société aux contraintes du marché capitalistique ni à la concurrence des sites de production, mais au contraire de l'organiser conformément aux valeurs et objectifs souhaités par la société et dotés d'une légitimité démocratique ». Guidé par cette vision, IG Metall plaide pour un Etat social qui offre à toutes les citoyennes et tous les citoyens les prestations de la prévoyance et de la protection sociales, mais attend d'eux en contrepartie qu'ils participent tous au financement de ces prestations. Telle est la vision fédératrice d'un Etat social plus équitable que défend notre syndicat. IG Metall ne prône donc pas le maintien de l'Etat social dans sa configuration actuelle, ni ne plaide pour un Etat social différent, et encore moins dans une version minimaliste. IG Metall revendique à l'inverse une politique coordonnée de changement progressif pour faire évoluer le modèle actuel vers un Etat social démocratique.

IG Metall s'oppose à la poursuite de la privatisation de l'Etat providence. Celleci est en contradiction avec la représentation d'un homo œconomicus qui serait capable, en ne mobilisant que ses ressources propres, d'organiser seul sa protection / prévoyance, et qui n'aurait de rêve plus cher que celui d'un Etat réduit a minima. Au contraire, IG Metall défend la vision rationaliste d'une prévoyance solidaire qui implique nécessairement le recours aux moyens et dispositifs de l'Etat social. Mais face aux mutations structurelles de la société, ce modèle requiert une solidarité nouvelle, c'est-à-dire renforcée, entre les citoyen(ne)s vivant en Allemagne et, en conséquence, une répartition nouvelle des charges, c'est-à-dire mieux équilibrée. II s'agit donc de trouver une nouvelle et meilleure articulation entre les capacités respectives des individus, de la société et de l'Etat. C'est ainsi que nous parviendrons à construire, dans un environnement qui a profondément changé, un Etat social performant et démocratique, qui soit capable d'apporter à tous sécurité et prévoyance et, ce faisant, de créer les conditions permettant de garantir l'avenir démocratique et économique de notre pays.

\section{Réorganisation du financement et développement des infrastructures sociales}

Un Etat social solidaire et équitable doit reposer sur deux piliers principaux. Le premier est la structure de son financement; elle doit être réaménagée. Même si les débats sur la forme concrète à lui donner sont loin d'être clos, il s'en dégage d'ores et déjà deux options fondamentales. D'une part, celle de l'extension du financement actuel des régimes de protection sociale vers les modèles
Responsabilité individuelle et protection collective

Pour une politique coordonnée de changement progressif

Nouvelle articulation des capacités entre individus, société et Etat

Financement : une assiette élargie 
notamment dans la formation

Plus de professionnalisme dans l'auto-administration des caisses d'une assurance citoyenne (Bürgerversicherung) ou d'une assurance des actifs (Erwerbstätigenversicherung) qui permettraient d'inclure dans le système solidaire de protection tous les types de revenus et toutes les catégories d'actifs, et non plus seulement les revenus salariés ou les seuls actifs occupés. D'autre part, l'option d'un financement plus largement fiscalisé des régimes de protection sociale, tout particulièrement dans les domaines relevant des missions collectives d'intérêt général.

Le second pilier est celui des prestations sociales. Toute réforme de la protection sociale devra poursuivre l'objectif de développer les investissements dans les infrastructures sociales, en donnant priorité aux services sociaux et à l'offre de formation. Car l'avenir de ces deux domaines - la formation et les soins aux personnes - ne se résume pas uniquement à des transactions financières. D'autres incitations, et surtout des infrastructures sociales de qualité, sont mieux à même de contribuer à l'intégration sociale comme à l'amélioration de la qualité des prestations.

\section{Plus d'autonomie administrative pour une meilleure politique sociale}

Si les syndicats ont un pouvoir d'influence sur le patronat et le monde politique, ils disposent aussi d'une arène propre pour influer, dans l'intérêt des assurés sociaux, sur l'amélioration de l'efficience de l'Etat social. Par tradition, l'autonomie administrative est le moyen de donner corps aux prestations concrètes des différents systèmes d'assurance sociale. Des milliers de syndicalistes assurent au quotidien le travail d'auto-administration des caisses. Leur travail consiste à répartir les moyens, à améliorer la qualité des prestations et à assumer la responsabilité d'aménager l'avenir d'une politique de protection sociale efficiente. Bien que l'autonomie administrative ait été quelque peu victime, au cours des dernières années, d'un certain affaiblissement voulu par le monde politique, les syndicats considèrent qu'elle continue de leur offrir un terrain favorable pour faire valoir leur influence dans l'intérêt des assurés sociaux. Toutefois, pour mieux mener à bien cette mission, il est indispensable d'accroître le professionnalisme des acteurs impliqués dans les instances d'autonomie administrative et de renforcer leur coopération avec IG Metall.

\section{Retraites : un mix sur la base d'un premier pilier renforcé}

Développer les retraites d'entreprise
Le démantèlement de l'assurance retraite légale a atteint un point de non retour qui remet aujourd'hui en question son efficience comme sa légitimité. Or la stratégie de politique sociale majoritairement préconisée, et qui consiste à reconfigurer l'assurance retraite sous la forme d'un mix reposant sur trois piliers, à savoir, dans l'ordre, du régime de l'assurance retraite légale, d'un régime d'entreprise et d'une assurance privée, ne peut déboucher sur un système viable qu'à une condition: que le premier pilier (l'assurance retraite légale) reste le socle de l'ensemble du système, et que le second bénéficie d'un soutien conséquent en comparaison du troisième. Les mesures engagées en la matière par le gouvernement fédéral sont jusqu'à présent insuffisantes. Pour leur part, les syndicats mettront tout en œuvre pour que l'assurance retraite légale reste au cœur de la protection vieillesse des Allemands et que le second pilier connaisse un développement conséquent grâce à un dispositif de soutien obligatoire abondé par des fonds publics et des cotisations patronales.

\section{Santé : assurance citoyenne solidaire + orientation qualité}

Alors que le niveau des dépenses de santé par habitant place l'Allemagne au troisième rang mondial, la qualité des prestations n'est pourtant que médiocre dans la plupart des domaines fondamentaux. Cela signifie concrètement que, en Allemagne, plus de gens meurent des suites d'un infarctus, d'une attaque cérébrale, d'un cancer ou d'un diabète que dans la plupart des autres Etats membres de l'UE. Le système allemand de santé et d'assurance dépendance 
souffre donc d'un double problème de financement et de qualité des prestations. Pourtant, à niveau de dépenses identique, il est possible d'assurer une meilleure qualité des soins et prestations.

Les débats actuels sur une réforme du système d'assurance maladie se concentrent sur l'avenir du financement des soins garantis par le régime public. Alors que le modèle de la prime forfait santé (Gesundheitsprämie) qui a la préférence de l'opposition et du patronat vise en premier lieu à alléger le poids des prélèvements pesant sur les entreprises, le modèle de l'assurance citoyenne solidaire que préconisent les syndicats est fondé sur une base financière à la fois plus équitable et plus rentable. IG Metall va plus loin encore en revendiquant un système d'assurance maladie intégré et avec une plus grande orientation qualité, afin de mieux mettre à profit les marges existantes pour gagner en efficience et en qualité tout en réalisant plus d'économies. II s'agit donc de mettre en oeuvre un financement plus équitable et une politique de santé plus efficace afin de tirer le meilleur parti de notre système de santé dans l'intérêt de tous. L'objectif d'une politique de santé solidaire est de veiller à ce que chacun ait accès, en cas de besoin, aux prestations médicales adaptées à son cas et socialement disponibles.

\section{Emploi :}

mesures de soutien public à l'emploi + soutien individualisé amélioré

Un chômage de masse incompressible et la hausse du chômage de longue durée révèlent les limites de la politique pour l'emploi. Celle-ci n'est pas en mesure, seule, de créer des emplois ; elle peut, au mieux, venir en complément des divers axes d'une politique macro-économique menée pour générer croissance et emplois. Cela étant, la restructuration de l'Agence fédérale pour l'emploi, qui vise à accroître le taux de placement des chômeurs, à individualiser le soutien et à proposer des mesures de (re)qualification adaptées, est une avancée importante pour améliorer la perméabilité entre chômage et travail. Mais tant que ne seront pas créés plus d'emplois, le nouveau dispositif de soutien ne pourra, à compter qu'il soit rapidement mis en place, produire que des effets limités. Si les tensions persistent sur le marché de l'emploi, il faudra alors développer la politique de soutien public à l'emploi. De même, il conviendra alors de remettre à plat la politique de réformes sociales menée jusqu'ici pour mieux l'adapter à l'évolution du contexte.

\section{Famille :}

\section{étendre et améliorer les infrastructures pour l'enfance et le 3ème âge}

Les dépenses de politique familiale de l'Allemagne sont parmi les plus élevées au monde. Pour des résultats plus que médiocres: une pauvreté en hausse chez les enfants, de faibles perspectives de formation pour les enfants issus de milieux défavorisés, de grandes difficultés à concilier vie familiale et professionnelle, sans parler de l'énorme incapacité du dispositif à faire face à un nombre croissant de personnes dépendantes. IG Metall revendique un engagement financier accru pour développer des infrastructures d'accueil performantes, pour aider les familles avec enfants et soutenir le maintien à domicile des personnes âgées, comme pour permettre aux femmes actives de mieux concilier vie familiale et professionnelle. Pour sa part, IG Metall mène ses propres programmes en la matière, qu'ils soient internes à l'entreprise ou inter-entreprises, et apporte sa contribution aux projets développées par le gouvernement fédéral comme le DGB, de l'échelon de l'entreprise à l'échelon national.

\section{Développer la politique sociale au niveau de l'entreprise comme de la branche}

Depuis quelques années, l'entreprise tend à prendre une importance nouvelle en tant que lieu d'initiatives en matière de politique sociale. Et cela, dans des
Réaliser des économies et des gains d'efficience

Des mesures en complément d'une politique macro-économique de croissance et d'emploi

L'entreprise a une responsabilité collective 
domaines aussi importants que la protection vieillesse, la formation continue ou la protection sociale du travail (santé et sécurité). Bien que l'entreprise ne puisse pas compenser la réduction des prestations de l'Etat social, elle n'en est pas moins un lieu autonome et responsable, capable de contribuer, en prenant les initiatives appropriées, à améliorer la qualité de vie, l'aptitude au travail et la santé des Allemands. Elle se doit, dans ce cadre, de prendre en considération tout particulièrement la situation des salariés âgés dont les effectifs ont baissé dramatiquement dans les entreprises au cours des dernières années. Pour inverser cette tendance, d'importants efforts sont nécessaires dans le domaine de la formation continue et de la promotion de la santé au niveau de l'entreprise. Le syndicat IG Metall a fait tout ce qui est en son pouvoir, grâce aux conventions collectives qu'il a conclues, pour rendre accessible à tous les salariés les régimes des retraites d'entreprise. Mais comme les résultats ne sont encore guère satisfaisants, il convient d'explorer de nouvelles voies pour instituer un système de retraites d'entreprise autonome et généralisé. IG Metall revendique donc de rendre obligatoire la participation du patronat et un engagement financier plus important de sa part.

\section{Développer le modèle social européen et combattre l'économicisation de la politique sociale allemande}

L'intégration européenne ne peut se réduire à la construction d'un marché communautaire. Il faut à l'Europe en cours de constitution un fondement social. Voilà pourquoi il convient de préserver le modèle social européen et de le développer dans le contexte de l'élargissement à l'Est pour l'adapter à la nouvelle situation. Mais ce projet est mis en danger par une politique trop exclusivement dédiée au Pacte de stabilité, et qui soumet la politique sociale nationale, allemande, à des impératifs de concurrence renforcée. Cela remet en question le caractère solidaire de nos systèmes nationaux de protection sociale.

Le Traité constitutionnel européen constitue un pilier important pour le développement d'un modèle social européen. II livre le cadre social qu'il revient aux syndicats de structurer pour lui donner vie. Le Traité constitutionnel pose en effet des jalons fondamentaux en ce qui concerne la reconnaissance des droits des salariés comme des prérogatives syndicales - autant d'aspects fondamentaux pour l'institution d'une Europe sociale. 